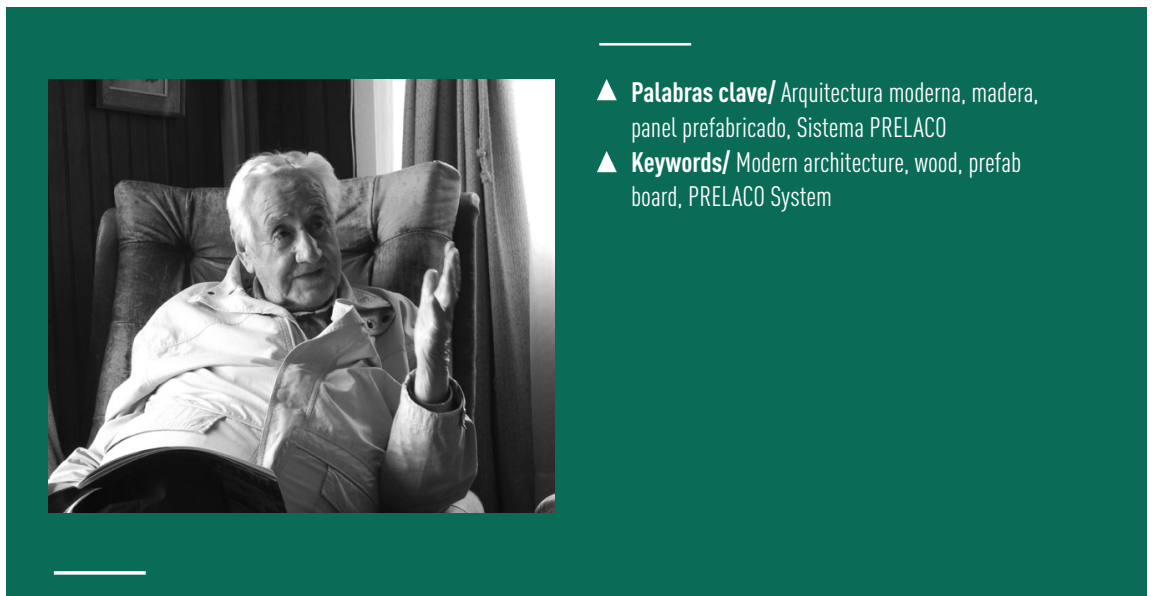

ENTREVISTA / INTERVIEW

\title{
Entrevista al arquitecto Néstor Holzapfel Gross.
}

\author{
Interview with architect \\ Néstor Holzapfel Gross.
}

\author{
Gonzalo Cerda-Brintrup \\ Arquitecto, Universidad del Bío-Bío, Chile. \\ Magister en Rehabilitación del Patrimonio \\ Arquitectónico y Urbano, Universidad Politécnica \\ de Madrid, España \\ Doctor en Arquitectura y Urbanismo, \\ Universidad del Bío-Bío, Chile. \\ Profesor asociado e investigador del Grupo de \\ Investigación en Patrimonio de la Universidad \\ del Bío-Bío, Chile. \\ gcerda@ubiobio.cl
}

\begin{abstract}
El arquitecto Néstor Holzapfel Gross' (Carahue, 1927) fue uno de los primeros arquitectos en titularse en la Universidad de Chile - en 1952 - bajo el nuevo régimen académico derivado de la Reforma Universitaria de 1944. Desarrolló en Puerto Montt y el sur de Chile una extensa vida profesional ligada a la arquitectura y posteriormente al turismo - hasta la actualidad - en donde puso en práctica los nacientes postulados de la Arquitectura Moderna, reinterpretada en el material constructivo del que disponía en la zona, la madera. A continuación, se transcribe parte de dos extensas entrevistas ( 5 de febrero de 2017 y 4 de junio de 2017) realizadas en Ensenada, Provincia de Llanquihue, donde vive actualmente. El arquitecto habla sobre su obra y el Sistema PRELACO, un panel prefabricado de madera con el que llegó a construir más de 100 escuelas en la región. / Architect Néstor Holzapfel Gross' (Carahue, 1927) was one of the first architects in graduating from the University of Chile - in 1952 - under the new academic scheme resulting from the University Reform of 1944. He conducted a long professional career in Puerto Montt and southern Chile, at first linked with architecture and then with tourism - up until today. He implemented the nascent tenants of Modern Architecture reinterpreted in the local building material available to him: wood. The text is a transcription of two extensive interviews (February 5, 2017 and June 4, 2017) conducted in Ensenada, Province of Llanquihue, where he lives today. Here, the architect speaks about his work and the PRELACO System, a prefabricated wooden board with which he has built more than 100 schools in the area.
\end{abstract}

\section{Néstor, ¿dónde estudió Ud. y cuando se tituló?}

Estudié en la Universidad de Chile entre 1946 y 1952; me recibí el año 1952. Yo provenía de Carahue, en La Araucanía; nuestra generación fue de las primeras en estudiar con el nuevo régimen instaurado después de la reforma de 1944.

\section{¿En qué consistió su proyecto de título?}

Consistió en una fábrica de laminados de placa de fibra. Por esos años yo ya estaba interesado en la producción industrial, en la prefabricación, y desarrollé un proyecto de título que estaba en la vanguardia de esos temas. Por esos años llegaron a Chile, traídos por la Corporación de Fomento de la Producción (CORFO), unos asesores de Finlandia, que venían a apoyar el desarrollo de una fábrica de placas de madera en Chile; fueron los años en que se formó la Fábrica Cholguan. Cuando los empresarios que estaban formando Cholguan supieron de mi proyecto, me lo solicitaron y con fotos de él promocionaron la futura industria; vendieron acciones con fotos de mi proyecto. Una vez que la fábrica se levantó, en retribución me regalaron 30.000 acciones.

A propósito de Finlandia, cera conocida en esos tiempos en Chile la obra de

\section{Alvaar Alto?}

Alvaar Alto en esos tiempos estaba de moda, representaba para nosotros la "escuela integral". 


\section{¿Cuándo se vino al sur?}

Me titulé el 28 de diciembre de 1952 e inmediatamente me casé y vine a Puerto Montt. Recuerdo que el profesor Oyarzún que era Director de Arquitectura del Ministerio de Obras Públicas (MOP), me ofreció ir a trabajar a lquique o Puerto Montt. Yo elegí Puerto Montt y me vine inmediatamente. Aquí llegué a trabajar como arquitecto ayudante de la Dirección de Arquitectura, pero duré solamente un año, no me gustó; ¿sabe cuál era el apodo que me tenían? El erizo. Era espinudo, no lograba adaptarme a lo que me pedían,

\section{tenía otras ideas.}

\section{¿Entonces, qué hizo?}

Bueno, formé mi propia oficina y comencé a desarrollar encargos y obras con la arquitectura que a mí me interesaba, pero a la gente no le gustó. A las personas no les gustaban mis obras, eran demasiado diferentes a lo que estaban acostumbrados. De hecho, hice muy pocas obras para particulares en Puerto Montt.

Ahí entonces me di cuenta que no podía continuar así, que debía hacer algo, y formé la industria PRELACO: Pre Elaboración de Elementos de la Construcción. Yo ya desde la escuela tenía el interés por la prefabricación y comencé a producir puertas y ventanas. La idea ya venía de mi proyecto de título y la de los prefabricados venía del Taller Central de la Chile. En el Taller Central se experimentaba con esas cosas, además que estábamos bajo los efectos del terremoto de Chillán.

Mire, ya desde los tiempos del Taller Central es que he pensado que la arquitectura es como la salud: sistémica, orgánica, funcional y armónica; es todo eso al mismo tiempo. Recuerdo que en la escuela nos venía a hacer clases un médico, el profesor García
Valenzuela, quien ya nos hablaba de esto. Bueno, volviendo a Puerto Montt, ahi formé PRELACO, compré maquinarias, un terreno, y me puse a producir elementos prefabricados en madera, seriados, y ahí surgió el "Panel Único PRELACO".

\section{¿En qué consistió el "Panel Único} PRELACO"?

Yo estaba obsesionado en aquellos tiempos con la producción en serie. Veía que los automóviles se producían en serie y me preguntaba ¿cómo sería posible producir edificios, viviendas, arquitectura en serie? ¿cómo hacer casas en un carril continuo? Ahí vino el gobierno de Frei Montalva y apareció con un plan para construir escuelas, un gran plan de gobierno para construir escuelas². Llegué ahí con mi chifladura y propuse mis primeros prefabricados y la Placa Única, que llamé "Panel Único PRELACO".

El panel estaba inspirado en una caja de bombones... ¿recuerda las cajas de bombones que uno le regalaba a su novia? bueno, de ahí viene la idea: de una tapa que calza perfecta con la otra. El panel tenía 2,80m de alto por 1,10m de ancho, o se podía hacer de la medida que uno quisiera, dependiendo de su función, ya que con el mismo panel se podían hacer muros, cielos rasos, aleros, frontones, pisos, entablados de cubierta... todo con un mismo panel. Tenían que ser livianos, que se los pudiera un hombre.

El panel tenía una estructura y se ensamblaba por caras. No tenía diagonales, ya que la rigidez la daba el machihembrado de terminación. Nosotros mismos fabricábamos el panel en PRELACO; la viruta que sobraba se mezclaba con cal y se ocupaba de relleno para la aislación térmica y acústica. El panel fue certificado por IDIEM, quien corroboró la calidad térmica y acústica de la placa.

Recuerdo que en una propuesta en el Ministerio de Educación, cuando aparecí con los planos del panel, misteriosamente desaparecieron las láminas con la que iba a presentarme. En ese momento el ministro Santa María, que era ministro y arquitecto al mismo tiempo, mandó a buscar y reponer las láminas, y las encontraron.

A partir de ese momento empecé a ganar casi todas las propuestas para el ministerio y la Sociedad Constructora de Establecimientos Educacionales. La placa única era barata, liviana, fácil de construir y de instalar, se colocaba rápido y se mejoraban los tiempos de construcción. Llegamos a construir más de 100 escuelas, acá en Llanquihue, Chaitén, Chiloé, Aysén. En nuestros mejores tiempos teníamos más de 300 personas trabajando en Puerto Montt; 200 en Aysén, mire, en un momento llegamos a tener 1.004 personas trabajando El año 1965 viajé a Noruega a una gran exposición de viviendas prefabricadas, buscando si había algo parecido a mi panel prefabricado, busqué y no encontré nada parecido.

\section{¿Cuánto tiempo funcionó la Fábrica PRELACO?}

Funcionó desde 1953 hasta 1970 aproximadamente. Recuerdo de esos años que el 73 me llevaron preso porque era amigo de Salvador Allende. Estando en la cárcel, el alcaide un día me vino a avisar que acababa de ganar el concurso para el proyecto del Museo Regional de Ancud en el

que estaba participando (se ríe).

\section{¿Tiene planos de esos paneles?}

Tendría que buscarlos, mucho se perdió en un incendio que tuvimos... (trae un maletín) pero tengo esto: hice estas maquetitas para explicar mi panel; con ellas iba a los diferentes ministerios y las diversas autoridades para explicar mi sistema. Muchas veces fui atendido por Edwin Weil como Director Nacional de Arquitectura del MOP. Les llevaba también este rompecabezas de madera que yo mismo fabriqué, mi panel PRELACO era como un rompecabezas, en el que todo ensamblaba perfectamente. 


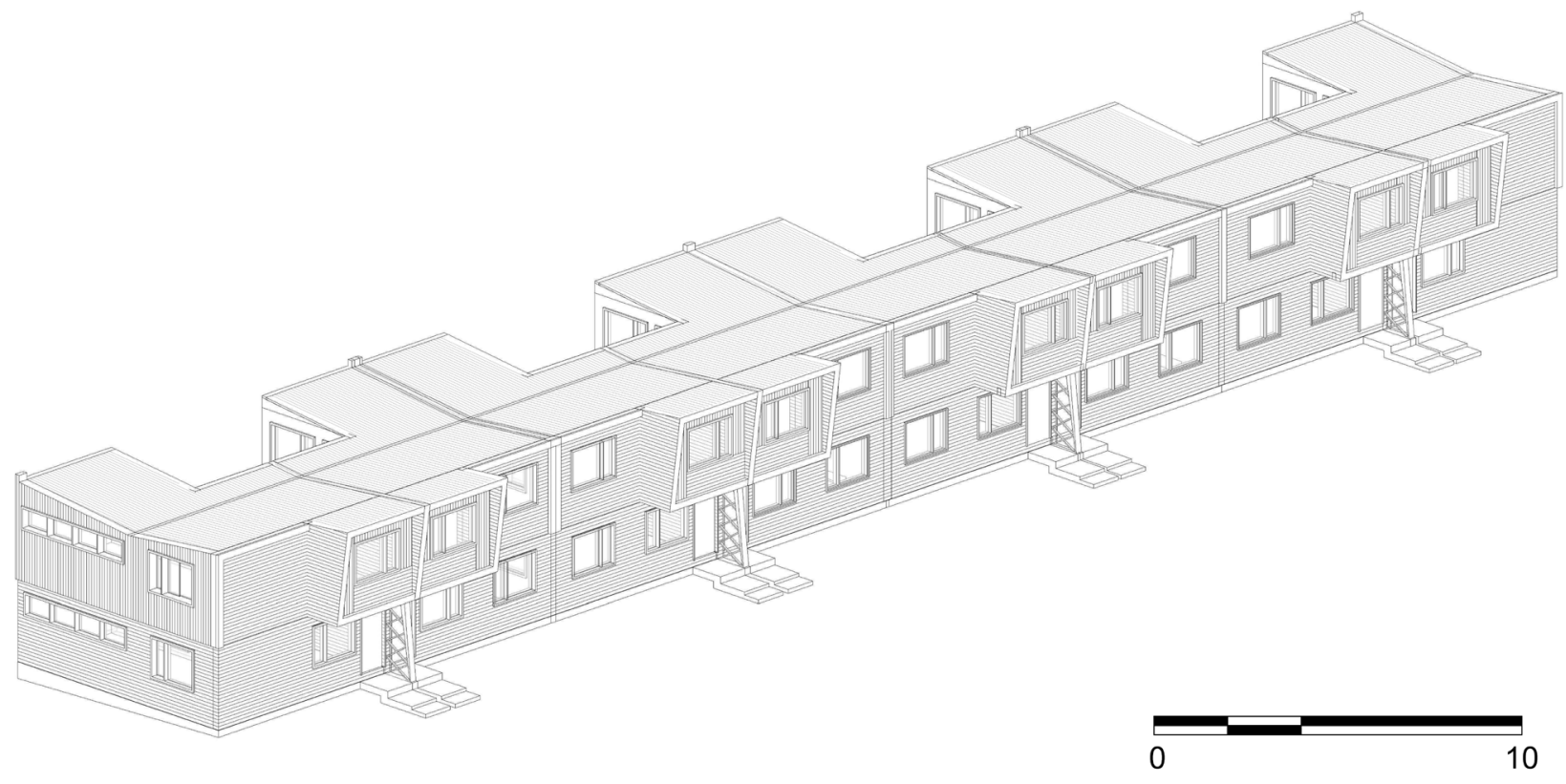

Figura 1. Isométrica Conjunto Urmeneta (fuente: Pablo Miranda C.)

\section{RESEÑA DE OBRAS}

Se reseña a continuación, 3 obras

proyectadas y construidas por el arquitecto Holzapfel en la Provincia de Llanquihue: El Conjunto Urmeneta de Puerto Montt (1962), la Casa Holzapfel en Puerto Montt (1965) y la Escuela Rural Río Pescado, camino a Ensenada (1965), uno de los más de 100 edificios escolares construidos con el Sistema PRELACO.
CONJUNTO URMENTA, Puerto Montt

Ubicación: Calle Urmeneta esq. Anibal Pinto, Puerto Montt; Región de Los Lagos.

Arquitecto: Néstor Holzapfel Gross

Fecha de construcción: 1962

$\mathbf{N}^{\circ}$ viviendas del conjunto: 6 viviendas Superficie viviendas: $66 \mathrm{~m}^{2}$

El Conjunto Urmeneta constituye uno de los primeros conjuntos de vivienda colectiva promovidos por un particular en Puerto Montt, y representó hacia la década de 1960 un modo innovador del vivir central, en una ciudad en donde lo regular era la vivienda unifamiliar aislada (figura 1)

Destaca en el desarrollo del conjunto y la vivienda su gran racionalidad y orden. Las áreas húmedas-cocinas y baños- se parean, tanto en primer como en segundo nivel, buscando racionalizar, ordenar y economizar en las instalaciones de alcantarillado y agua potable (figura 2).

Un elemento que destaca la absoluta racionalidad en la resolución de la planta - muy en concordancia con los nuevos postulados de la Arquitectura Moderna - es la reducción al mínimo de las circulaciones interiores, tanto en el primer como en el segundo piso. A excepción de la escalera, no existen pasillos, lo que no impide una circulación claramente resuelta, con accesos independientes para cada recinto.

La volumetría y expresión arquitectónica del conjunto apela a un orden geométrico y abstracto. La cubierta se resuelve mediante un "techo mariposa", en boga en la década de 1960, el que reemplaza la tradicional cumbrera superior del techo, por una limahoya que recoge las aguas en una línea y canal central (figura 3). 
CONJUNTO URMENETA

PUERTO MONTT
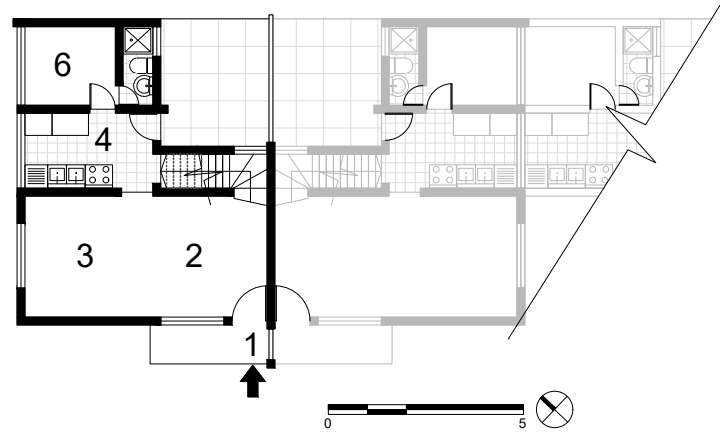

PRIMER NIVEL

1- ACCESO

2- ESTAR

3- COMEDOR

4- BANIO

6- DARMITORIO

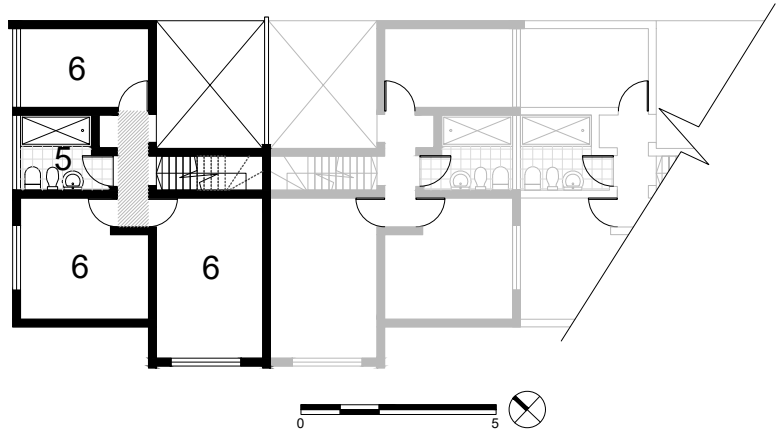

SEGUNDO NIVEL

Figura 2. Plantas Conjunto Urmeneta (fuente: Dibujo Anabella Benavides V. sobre un original de Jorge Lobos C.).

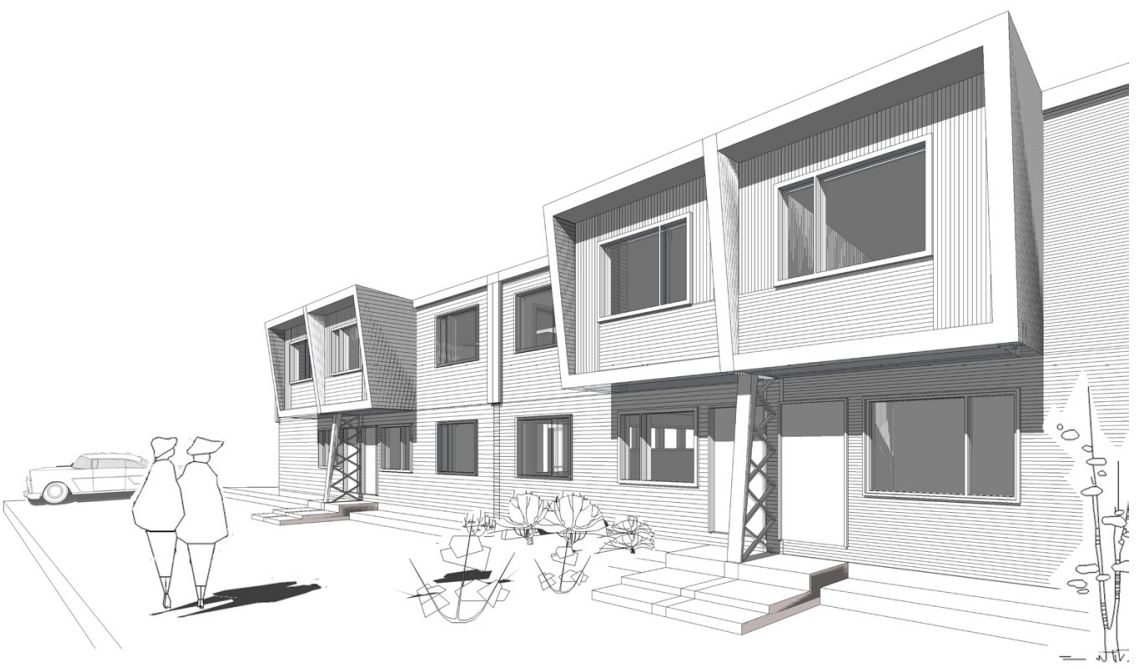

Figura 3. Pespectiva Conjunto Urmeneta (fuente: Pablo Miranda C.) 


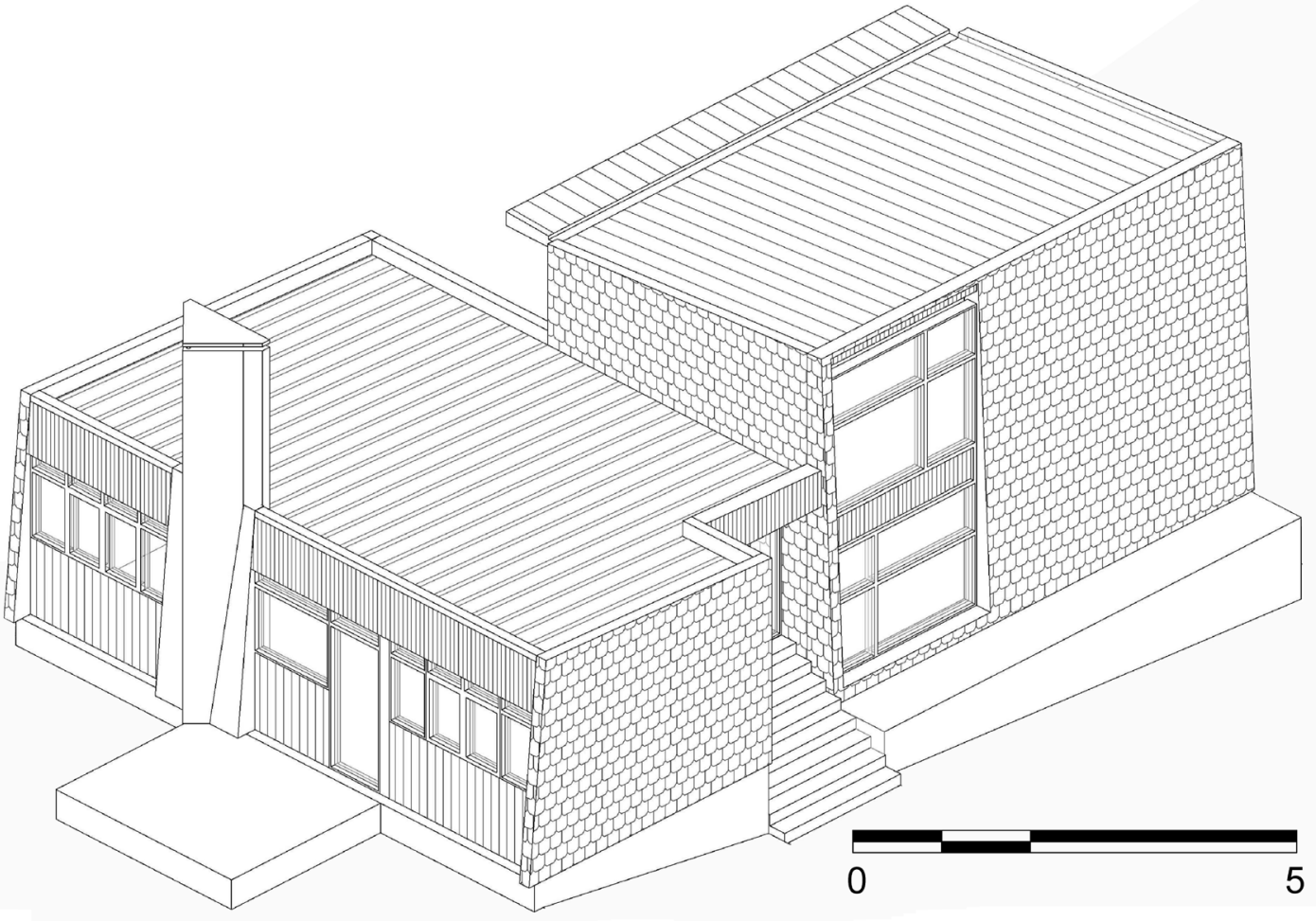

Figura 4. Isométrica Casa Holzapfel, Puerto Montt (fuente: Pablo Miranda C.).

\section{CASA HOLZAPFEL, Puerto Montt}

Ubicación: Calle Benavente esquina Aníbal Pinto, Puerto Montt. Región de Los Lagos.

Fecha de construcción: 1965.

Arquitecto: Néstor Holzapfel Gross Superficie construida: $133 \mathrm{~m}^{2}$

Es posible situar a esta obra dentro de la tendencia denominada por el arquitecto y crítico italiano Bruno Zevi como NeoExpresionista (Zevi, 1980), es decir, un período de la Arquitectura Moderna del siglo XX en que se busca romper con la ortodoxia funcionalista y la arquitectura resuelta volumétricamente como paralelepipedos rectos que se yuxtaponen, en la que se experimenta con mayor libertad en las formas (figura 4).

El arquitecto Ramón Méndez se ha referido a la influencia del Neo-Expresionismo en el desarrollo de la arquitectura chilena de la década de 1960 (Méndez, 1981), como lo hacen también Humberto Eliash, Manuel Moreno (Eliash, Moreno, 1989) y Pablo Fuentes (Fuentes, 2008) planteando que corresponde a un período en que se ensaya una arquitectura de mayor libertad formal dando un paso más allá de la estricta geometría racionalista (figura 5). Conforme lo indicado por su autor, su arquitectura fue incomprendida en una ciudad y un entorno social acostumbrado a otro tipo de obras. Su rupturismo y vanguardia sin embargo son reconocidos hoy, en una obra arquitectónica que con fuerza innovadora reinterpretó en madera las nuevas claves de la modernidad arquitectónica. La vivienda referida representa hoy una de las obras fundamentales de la Arquitectura Moderna en madera en el sur de Chile (figura 6). 


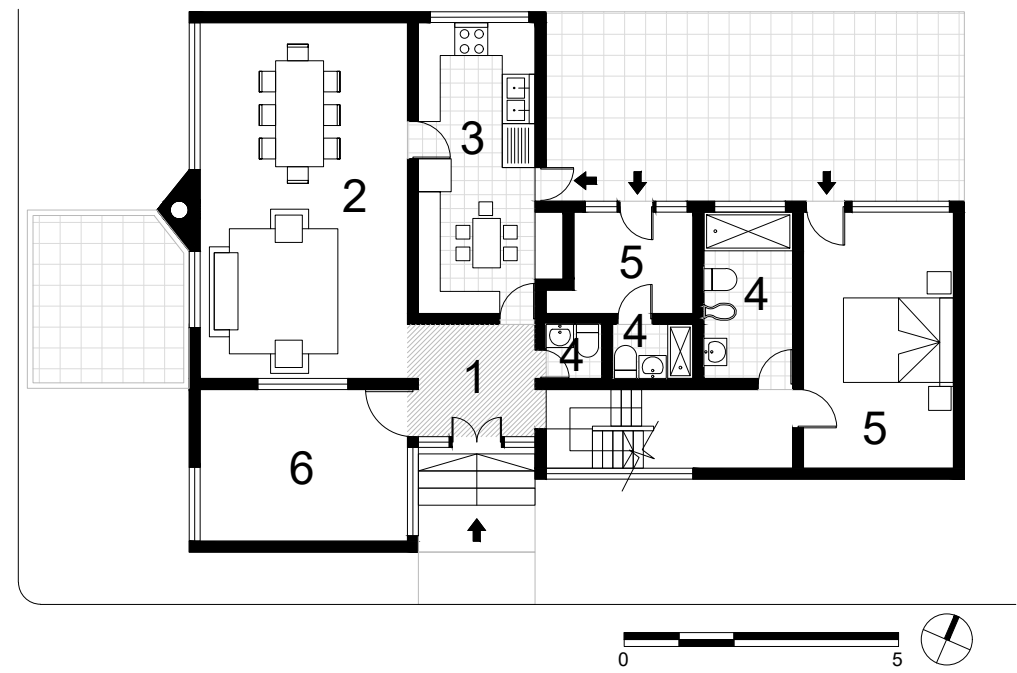

1- VESTÍBULO

2- ESTAR - COMEDOR

3- COCINA - COMEDOR DIARIO

4- BAÑO

5- DORMITORIO

6- ESCRITORIO

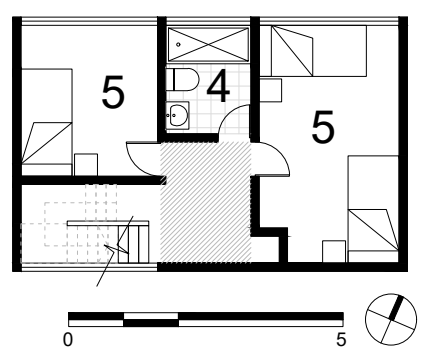

Figura 5. Plantas Casa Holzapfel (fuente: Dibujo Anabella Benavides V. sobre un original de Jorge Lobos C.).

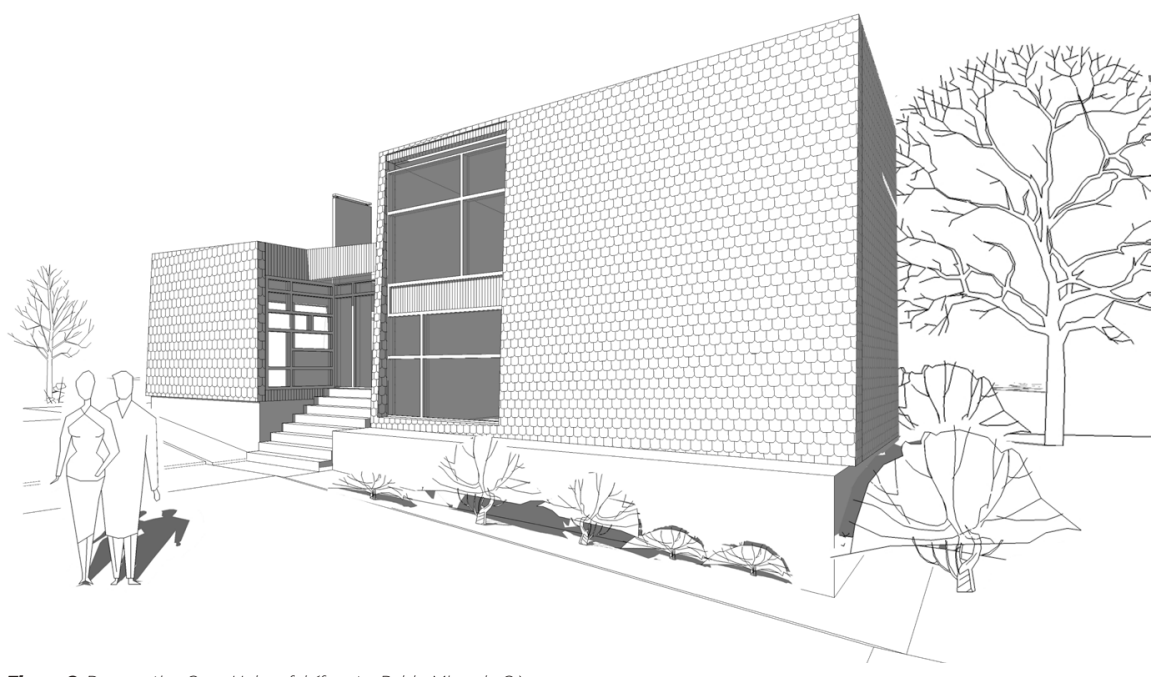

Figura 6. Perspectiva Casa Holzapfel (fuente: Pablo Miranda C.) 


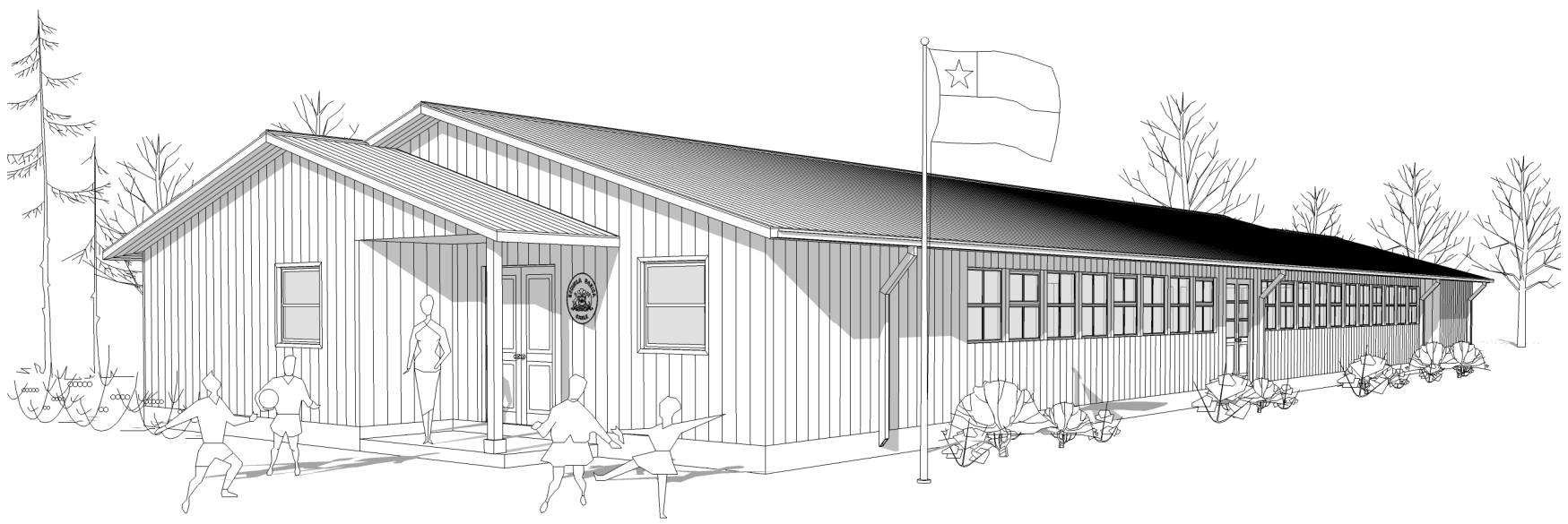

Figura 7. Perspectiva Escuela Rio Pescado (fuente: Claudio Monje M.)

ESCUELA RURAL RIO PESCADO

Ubicación: Rio Pescado; km. 17,5 camino

Puerto Varas-Ensenada; Provincia de

Llanquihue; Región de Los Lagos.

Arquitecto: Néstor Holzapfel Gross.

Fecha de Construcción: 1965

Sistema constructivo: Panel Único

\section{PRELACO}

Superficie construida: $353 \mathrm{~m}^{2}$

Este edificio debe comprenderse en la lógica de la acción constructiva de la Sociedad Constructora de Establecimientos Educacionales. La SCEE fue un organismo del Estado creado el año 1937 (Ley $N^{\circ}$ 5.989), destinado a desarrollar la infraestructura educacional que el país precisaba, en el ámbito de la enseñanza escolar, superior y técnico profesional (figura 7).

La SCEE propicia el desarrollo de equipamiento para la educación científicohumanista, como también para la formación a través de liceos agrícolas, escuelas normales, liceos comerciales y técnicos. La Sociedad construye establecimientos educacionales para la Enseñanza Elemental (escuelas básicas y rurales "tipo");
Enseñanza Superior (Escuelas Superiores y Grupos Escolares) y Enseñanza Vocacional (Liceos Técnicos y Liceos CientíficoHumanistas) (SCEE, 1987).

Un eslabón poco conocido de esta intensa producción arquitectónica y constructiva es el desarrollo de proyectos de escuelas en madera. En el sur del país la SCEE desarrolló un extenso programa de construcción de escuelas en madera, en especial Escuelas Básicas Elementales y Escuelas Rurales Tipo De tal modo, y siguiendo los prototipos desarrollados especialmente en la zona central del país, en la región sur acude al material disponible - la madera - y a la cultura constructiva del lugar, para desarrollar su obra.

El programa de una Escuela Básica Elemental y una Escuela Rural incluye: salas de clases, baños, una pequeña biblioteca, como asimismo siempre, un comedor con una cocina. Debe entenderse las escuelas rurales como una extensión del propio hogar, en el que se imparte no solo instrucción, sino constituyen el principal punto de encuentro y sociabilidad de niños y jóvenes que viven en el campo (imagen 1).

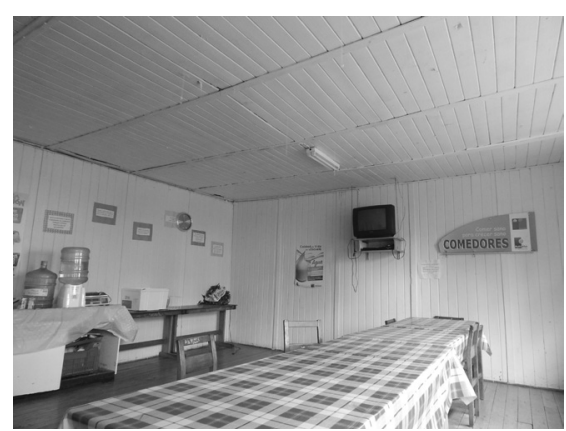

Imagen 1. Comedor Escuela Rio Pescado con Panel PRELACO (fuente: G. Cerda B.). 
LA OBRA DE NESTOR HOLZAPFEL EN EL SUR DE CHILE.

representa uno de los puntos más altos

de la Arquitectura Moderna en madera

en el sur de Chile y se le debe asociar a la producción arquitectónica desarrollada por profesionales que, no obstante, lejos de la

capital, hicieron una invaluable y silenciosa

contribución al desarrollo de la arquitectura chilena. Arquitectos como Néstor Holzapfe Gross; Miguel García Fernández; Javier Anwandter; Horst Baumann; Ewald Worner $\mathrm{K}$; Eugenio Ringeling junto a otros de mayor reconocimiento como Carlos Buschmann; Edwin Weil y Emilio Duhart Harosteguy, por nombrar solo algunos, dejaron su impronta arquitectónica en las ciudades del sur del país. $\mathbf{\Delta} \mathbf{0}$

\section{REFERENCIAS}

Eliash, H. y Moreno, M., 1989. Arquitectura y Modernidad en Chile, 1925-1965. Una realidad múltiple. Santiago: Ediciones Universidad Católica de Chile

Fuentes, P., 2008. El Desarrollo de la Arquitectura Moderna en Chile, 1929-1970: Apropiación, Debate y

Producción Arquitectónica. Vol. 1, p.755. Tesis doctoral Universidad Politécnica de Madrid.

Mendez, R. "Chile" en Fuentes, P. Op.cit.p.754
SCEE, 1987. Sociedad Constructora de Establecimientos Educacionales, 50 años de labor: 1937-1987. Santiago de Chile: Edición SCEE.

Torres Gilles, C., Valdivia Avila, S. y Atira Lemataire, M., 2015. Arquitectura escolar pública como patrimonio moderno de Chile. Santiago de Chile: Facultad de Arquitectura y Urbanismo Universidad de Chile; DOCOMOMO Chile; Consejo Nacional de la Cultura y las Artes.

Zevi. B., 1980. Historia de la Arquitectura Moderna. Barcelona: Ediciones Poseidón. 